\title{
The effect of food timing on fat oxidation during exercise and resting recovery
}

\author{
M. Honnor, M. Herdsman and A. L. Collins \\ Department of Nutrition \& Metabolism, Faculty of Health and Medical Sciences, University of Surrey, Guildford, \\ GU2 $7 X H, U K$
}

High levels of obesity and inactivity are major concerns to public health in the UK. With increasingly busy lifestyles and a lack of leisure time ${ }^{(1)}$, there is a demand for recommendations regarding the most effective nutritional strategy around exercise for maximal fat oxidation. This study aimed to compare the effects of high intensity interval training (HIIT) with carbohydrate feeding before or after exercise on resting, exercise, and post-exercise substrate utilisation.

Ten healthy untrained females (age 18-22 yr; BMI 22[SD $\left.0.73 \mathrm{~kg} / \mathrm{m}^{2}\right]$ ) performed a $\mathrm{VO}_{2}$ peak test and were randomly assigned to two treatment groups; CHO Pre $(n=5)$ and CHO Post $(n=5)$. Both groups completed two weeks of HIIT $(3$ day/wk, $8-10 \times 60$ second cycling bouts at $95 \% \mathrm{VO}_{2}$ peak separated by 90 seconds recovery at 50 watts) in which carbohydrate ( $1 \mathrm{~g} / \mathrm{kg}$ body weight maltodextrin) was consumed either prior to (CHO Pre) or post (CHO Post) exercise. Before and after the training period, substrate utilisation during 20 minutes at rest was determined via indirect calorimetry after an overnight fast (12 hours). One hour post a standardised breakfast $(336 \mathrm{Kcal}, 59 \mathrm{~g} \mathrm{CHO})$, substrate utilisation was measured during and for 2 hours after a 25 minute cycling bout at $50 \% \mathrm{VO}_{2}$ peak. Ethical approval for this study was obtained from the Faculty of Health and Medical Sciences at the University of Surrey.

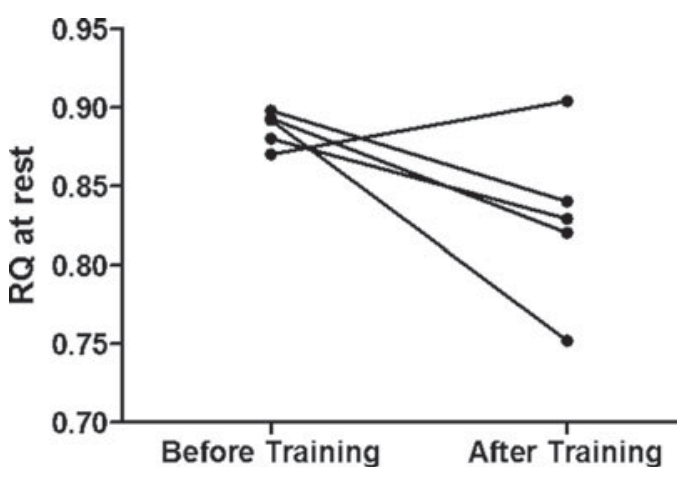

Fig. 1. Change in resting RQ with training in $\mathrm{CHO}$ Pre $(n=5)$.

Whilst not statistically significant, paired samples t-tests showed a trend towards a decrease in respiratory quotient (RQ) (see Figure 1) and increased fat oxidation $(\mathrm{g} / \mathrm{hr})$ at rest in CHO Pre $(p=0.090$ and 0.088 respectively), but not CHO Post $(p \geq 0.532)$ (Table 1$)$ was observed. Mean respiratory exchange ratio/RQ and fat oxidation $(\mathrm{g} / \mathrm{hr})$ during exercise and over 2 hours post-exercise were unaffected by the timing of carbohydrate $(p \geq 0.197)$. However there was a trend towards a decrease in RQ at each time point post-exercise $(p=0.056)$.

Table 1. Measured rates of fat oxidation pre and post training for both intervention groups (Mean and SE)

\begin{tabular}{|c|c|c|c|c|c|c|c|c|}
\hline \multirow[b]{3}{*}{ Fat oxidation $(\mathrm{g} / \mathrm{hr})$} & \multicolumn{4}{|c|}{ CHO Pre $(n=5)$} & \multicolumn{4}{|c|}{ CHO Post $(n=5)$} \\
\hline & \multicolumn{2}{|c|}{ Before Training } & \multicolumn{2}{|c|}{ Post Training } & \multicolumn{2}{|c|}{ Before Training } & \multicolumn{2}{|c|}{ Post Training } \\
\hline & Mean & $\mathrm{SE}$ & Mean & SE & Mean & $\mathrm{SE}$ & Mean & $\mathrm{SE}$ \\
\hline At Rest & 2.04 & 0.13 & 3.18 & 0.51 & 1.92 & 0.92 & 1.90 & 0.59 \\
\hline During Exercise & 1.60 & 0.60 & 1.8 & 0.58 & 2.26 & 0.40 & 2.50 & 0.36 \\
\hline During 2 hour recovery & 2.74 & 0.50 & 3.47 & 0.67 & 2.22 & 0.42 & 2.48 & 0.38 \\
\hline
\end{tabular}

Our findings failed to show a significant effect of the timing of carbohydrate around exercise training on fat oxidation either at rest, during, or post-exercise, possibly attributable to a small sample size. However, trends observed supprt the findings of Fuchs and Young ${ }^{(2)}$ suggesting that, in women, consuming carbohydrate before exercise may potentially be more beneficial for fat oxidation than consuming carbohydrate post-exercise. Larger placebo-controlled trials may have better potential to fully elucidate optimal eating strategies around exercise.

1. Murray, L. (2006) Sport, Exercise and Physical Activity: Public Participation, Barriers and Attitudes [Homepage of Information and Analytical Services Division], [Online]. Available at: http://www.scotland.gov.uk/Resource/Doc/932/0041468.pdf [Accessed March 2012].

2. Fuchs, A. \& Young, H. (2011) Investigation into gender differences in the effects of feeding around exercise on exercise performance, energy expenditure and substrate utilisation Proc Nutr Soc - In Press. 